\title{
Isolation, Identification, and Antibiotic Susceptibility Patterns of Pseudomonas aeruginosa Strains from Various Clinical Samples in a University Hospital in Northern Cyprus
}

\author{
Nadeem Ullah' (D), Emrah Güler² (1), Meryem Güvenir ${ }^{3}$ (D), Ayșe Arıkan² (D, Kaya Süer ${ }^{4}$ (1) \\ 'Department of Pathogenic Biology, Huazhong University of Science and Technology, Tongii Medical Collage, Wuhan, China \\ ${ }^{2}$ Department of Medical Microbiology, Near East University School of Medicine, Lefkoșa, Cyprus \\ ${ }^{3}$ Department of Microbiology, Near East University Vocational School of Health Services, Lefkoșa, Cyprus \\ ${ }^{4}$ Department of Infectious Diseases and Clinical Microbiology, Near East University School of Medicine, Lefkoșa, Cyprus
}

ORCID IDs of the authors: N.U. 0000-0003-2624-2393; E.G. 0000-0002-1635-005I; M.G. 0000-0002-9702-9947; A.A. 0000-0003-19421203; K.S. 0000-0002-2565-3425

Cite this article as: Ullah N, Güler E, Güvenir M, Arıkan A, Süer K. Isolation, Identification, and Antibiotic Susceptibility Patterns of Pseudomonas aeruginosa Strains from Various Clinical Samples in a University Hospital in Northern Cyprus. Cyprus J Med Sci 2019; 4(3): 225-8.

\section{BACKGROUND/AIMS}

Pseudomonas aeruginosa is a severe opportunistic bacterium responsible for frequently lethal nosocomial infections. It is highly resistant to commonly used antibiotics, creating a real challenge in the treatment of nosocomial infections.

\section{MATERIALS and METHODS}

A total of I52 samples were collected from various departments of the hospital, which included wound, blood, urine, cerebrospinal fluid (CSF), sputum, aspiration fluid, and ear, nose, and throat swab samples. The susceptibility pattern of bacteria was determined by using the BD Phoenix 100 automated microbiology system. P. aeruginosa was also identified according to its cultural, microscopic, morphological, and biochemical characteristics.

\section{RESULTS}

Samples were identified by using BD Phoenix 100 and conventional biochemical tests are used for confirmation. The results of antimicrobial susceptibility testing indicated that $P$. aeruginosa was sensitive to piperacillin-tazobactam (92.5\%), meropenem (87.2\%), colistin (86.7\%), ticarcillin-clavulanate (86.6\%), amikacin (81.5\%), imipenem (80.8\%), norfloxacin $(79.5 \%)$, cefepime (78.4\%), ceftazidime (76.0\%), gentamicin (76.0\%), levofloxacin (73.5\%), and ciprofloxacin (73.2\%). On the other hand, the resistance rates of amoxicillin, ampicillin-sulbactam, cefuroxime, nitrofurantoin, cefoxitin, trimethoprim/sulfamethoxazole, and ceftriaxone were found to be $100 \%, 98.7 \%, 97.7 \%, 97.7 \%, 94.7 \%, 94.7 \%$, and $93.8 \%$ respectively. The prevalence of antibiotic susceptibility of $P$. aeruginosa in Cyprus is more in males (62.5\%) than in females (37.5\%).

\section{CONCLUSION}

Knowledge about local antimicrobial resistance patterns of $P$. aeruginosa is essential for the guidance of empirical therapy by physicians, medical microbiologists, and public health officials. Antimicrobial surveillance should be done periodically to detect current resistance in our local area.

Keywords: Antibiotic susceptibility, Northern Cyprus, pseudomonas aeruginosa, resistance

\section{INTRODUCTION}

Pseudomonas aeruginosa is a gram-negative, bacillus, and non-spore forming bacterium. It is widely distributed in nature including soil, water, and various types of vegetation throughout the world (I, 2). It causes community-acquired and nosocomial infections such as pneumonia, urinary tract infections, and bacteremia. The infections can be particularly important in patients who are immunocompromized, such as neutropenic or cancer patients $(3,4)$. Nowadays, the rates of morbidity and mortality have been increased because of multidrug-resistant $P$. aeruginosa strains (5).

P. aeruginosa has an intrinsic and acquired resistance against many antibiotics. In addition, it can also gain resistance due to abusive or misuse of commonly used antibiotics (6). The microorganism possesses a natural resistance to antibiotics 
including aminoglycosides, cephalosporins, fluoroquinolones, and penicillins (7). This organism is the most common etiological agent of pneumonia, urinary tract infections, and in the bloodstream (8).

These bacteria have combination resistance mechanisms according to their cephalosporins production, having efflux pumps, low intrinsic outer membrane permeability, and target mutations. Because of this situation, in combination with the misuse of drugs, hospital infections of multidrug-resistant $P$. aeruginosa cause serious problems (9). Clinical isolates of gram-negative rods including $P$. aeruginosa have been shown to produce an enzyme called inducible beta-lactamases, which is the mechanism of bacterial resistance to beta-lactam antibiotics. These enzymes are plasmid-mediated (4). P. aeruginosa is isolated from various body fluids including sputum, urine, wounds, eye, ear, and blood. A narrow class of antibiotics is effective against $P$. aeruginosa, including the carboxypenicillins, quinolones, cephalosporins, and aminoglycosides $(9,10)$. As the antibiotic resistance profiles of $P$. aeruginosa can change in years, prevalence studies must be carried out regularly. The aim of this study was to determine the antibiotic susceptibility of $P$. aeruginosa from clinical samples and to contribute the application of appropriate empiric therapy in the Near East University in Northern Cyprus.

\section{MATERIALS and METHODS}

\section{Collection of Samples}

This study was carried out at our microbiology laboratory between June 2014 and October 2015. A total of 152 samples were collected from the different units of the hospital. These samples included urine, blood, nasal swab, sputum, aspiration fluids, intravascular catheters, CSF, and wound. All the samples were first processed to get a pure culture by subculturing using general and selective media as blood and eosin methylene blue agar. The demographic information was obtained from the hospital systems. The sensitivity pattern of the bacteria was determined by using an automated microbiology system (Becton-Dickinson, USA) according to the standards of Clinical $\bar{\alpha}$ Laboratory Standards Institute. Ethics committee approval was not taken due to in vitro design of the study. This study does not include human participants. Thus, no consent form was required.

\section{Statistical Analysis}

Statistical Package for the Social Sciences version 22.0 (SPSS IBM Corp.; Armonk, NY, USA) was used for the analysis of the data.

\section{RESULTS}

A total of 152 records were identified as $P$. aeruginosa from different specimens and were mostly isolated from urine samples in this study (Figure I). In our study, $62.5 \%$ of the subjects were males and $37.5 \%$ were females. Their ages ranged from 10 to 67 years and are presented in Figure 2. When the age distribution was considered, $P$. aeruginosa infections were more frequently seen in the ages between 10 and 20 and over 60. The antimicrobial susceptibility test revealed that $P$. aeruginosa was highly sensitive to most of the antibiotics tested. Piperacillin-tazobactam was the most effective drug against all $P$. aeruginosa $(92.5 \%$ sensitivity) followed by meropenem ( $87.2 \%)$, colistin $(86.7 \%)$, ti- carcillin/clavulanate (86.6\%), amikacin (81.5\%), and imipenem (80.8\%). On the other hand, P. aeruginosa had $98.7 \%$ resistance to ampicillin/sulbactam antibiotic and the other high resistance rates were for cefoxitin (94.7\%), trimethoprim/sulfamethoxazole (94.7\%), and ceftriaxone (93.8\%) (Table I).

\section{DISCUSSION}

According to the scientific studies, multidrug-resistant strains for both the hospitals and community constitute a serious problem, especially infections, induced by $P$. aeruginosa across the world (4). $P$. aeruginosa is a nosocomial bacterium, which is present in disinfectants, respiratory equipment, sinks, taps, and mops in the hospital by forming a biofilm (I). A total of I52 records were identified as $P$. aeruginosa from different specimens and were mostly isolated from urine samples in this study (Figure I). Regarding gender, $62.5 \%$ of the subjects were males whereas $37.5 \%$ were females. It is reported that infections caused by $P$. aeruginosa are more common in males than females. This is comparable with other studies that detected $61 \%$ of pseudomonas infections in males (II).

The ages of the patients ranged from 10 to 67 years (Figure 2). When the age distribution was considered, $P$. aeruginosa infections were more frequently seen in the ages between 10-20 and over 60 years. Our results are likely similar to other studies that

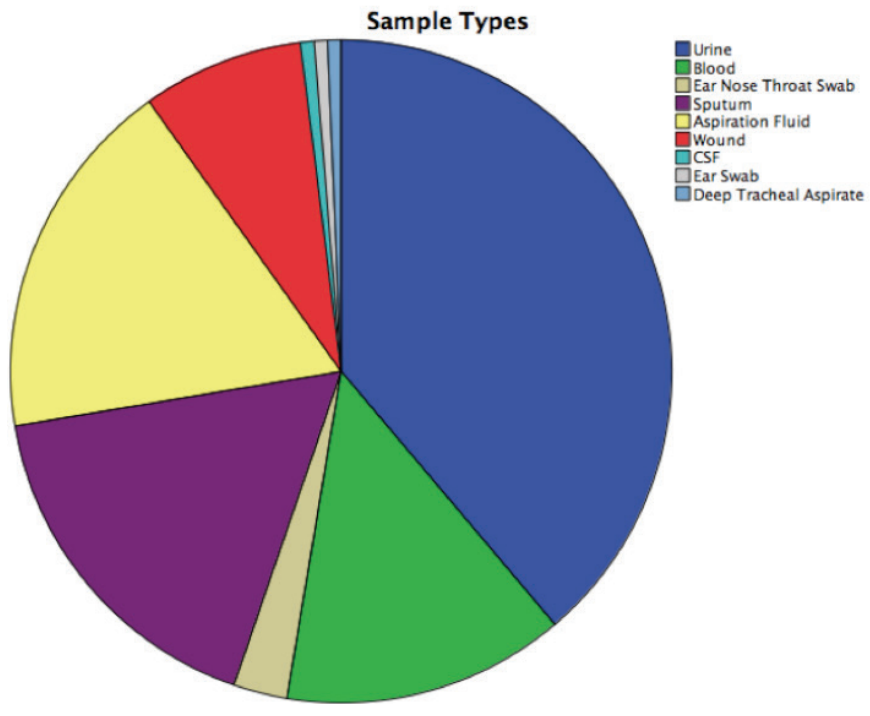

Figure I. Distribution of $P$. aeruginosa among different samples

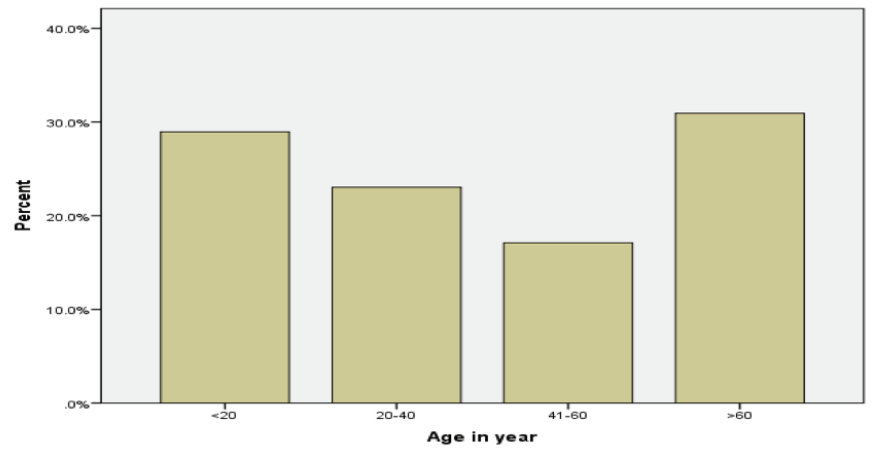

Figure 2. P. aeruginosa isolates from different age groups 


\begin{tabular}{|c|c|c|c|c|}
\hline Drugs & Total & $\begin{array}{l}\text { Sensitive } \\
\text { Count (\%) }\end{array}$ & $\begin{array}{l}\text { Intermediate } \\
\text { Count }(\%)\end{array}$ & $\begin{array}{l}\text { Resistant } \\
\text { Count (\%) }\end{array}$ \\
\hline Amikacin & 151 & $123(81.5)$ & $8(5.3)$ & $20(13.2)$ \\
\hline Ampicillin/Sulbactam & 151 & $2(1.3)$ & - & $149(98.7)$ \\
\hline Aztreonam & 152 & $76(50.0)$ & $18(11.8)$ & $58(38.2)$ \\
\hline Cefepime & 148 & $116(78.4)$ & $19(12.8)$ & $13(8.8)$ \\
\hline Cefoxitin & $15 \mid$ & $8(5.3)$ & - & $143(94.7)$ \\
\hline Ceftazidime & 150 & $114(76.0)$ & $5(3.3)$ & 31 (20.7) \\
\hline Ceftriaxone & $\| 3$ & $7(6.2)$ & - & $106(93.8)$ \\
\hline Ciprofloxacin & 149 & $109(73.2)$ & $5(3.4)$ & $35(23.5)$ \\
\hline Colistin & 113 & $98(86.7)$ & - & $15(13.3)$ \\
\hline Gentamicin & 146 & III (76.0) & $8(5.5)$ & $27(18.5)$ \\
\hline Levofloxacin & 113 & $83(73.5)$ & $4(3.5)$ & $26(23.0)$ \\
\hline Imipenem & 151 & $122(80.8)$ & $7(4.6)$ & $22(14.6)$ \\
\hline Meropenem & 149 & $130(87.2)$ & $6(4.0)$ & $13(8.7)$ \\
\hline Piperacillin/Tazobactam & 148 & $135(92.5)$ & - & $17(11.5)$ \\
\hline Ticarcillin/ Clavulanate & 144 & $64(86.6)$ & - & $46(42.6)$ \\
\hline $\begin{array}{l}\text { Trimethoprim/ } \\
\text { Sulfamethoxazole }\end{array}$ & 150 & $7(4.7)$ & $\mathrm{I}(.7)$ & $142(94.7)$ \\
\hline
\end{tabular}

found $43.5 \%$. aeruginosa infection in the age range of $\mathrm{I}-15$ years. Studies reported that incidence of $P$. aeruginosa increased with age and was higher in males than females (II, I2). Ak et al. (6) reported that the highest resistance was observed for levofloxacin (13\%) whereas the lowest resistance was observed for amikacin (3\%) in Malatya. Aykan and Çiftçi (I3) investigated the antibiotic resistance of $P$. aeruginosa isolates over II years (2003-2013) in Turkey. They reported that carbapenem resistance like imipenem resistance increased between 2007 and 2009; otherwise, these changes were not statistically significant for imipenem or meropenem. Resistance to aminoglycoside antibiotics such as amikacin, gentamicin, netilmicin, and tobramycin was decreased, but these changes were not found statistically significant. These study groups concluded that the antibiotic resistance in P. aeruginosa has started to decrease in Turkey.

In our study, the antimicrobial susceptibility test revealed that $P$. aeruginosa was highly sensitive to most of the antibiotics tested. Piperacillin/tazobactam was the most effective drug against all P. aeruginosa strains ( $92.5 \%$ sensitivity) followed by meropenem (87.2\%), colistin (86.7\%), ticarcillin/clavulanate (86.6\%), amikacin (81.5\%), and imipenem (80.8\%). On the other hand, P. aeruginosa had $98.7 \%$ resistance to the antibiotic ampicillin/sulbactam and the other high resistance rates were observed for cefoxitin (94.7\%), trimethoprim/sulfamethoxazole (94.7\%), and ceftriaxone (93.8\%) (Table I). Ruh et al. (14) studied the antibiotic resistance of $P$. aeruginosa between 2010 and 2014 in Northern Cyprus and reported that the resistance rate for aztreonam was $42.9 \%$ and susceptibility rate for levofloxacin was $79.8 \%$. According to our results, the resistance rate for aztreonam (38.2\%) and the susceptibility rate for levofloxacin (73.5\%) decreased in a one year period (2014-2015) in Northern Cyprus. In the present study, aminoglycoside (gentamicin 18.5\%; amikacin 13.2\%), fluoroquinolone (ciprofloxacin 23.5\%; levofloxacin 23\%), and ceftazidime (20.7\%) resistance among $P$. aeruginosa isolates were found to be higher with the rates reported in the antimicrobial resistance report of Southern Cyprus between 2012 and 2015 (I5).

The present study shows that the clinical isolates of $P$. aeruginosa are resistant to commonly used antibiotics and achieve more resistance to the newest antibiotics. In Cyprus, piperacillin/tazobactam, and meropenem are the most effective agents against $P$. aeruginosa whereas ampicillin/sulbactam, cefoxitin, and trimethoprim/sulfamethoxazole have the highest resistance. The knowledge of local antimicrobial resistance patterns is necessary to guide empirical therapy applied by practicing physicians, medical microbiologists, and public health officials.

Ethics Committee Approval: Ethics committee approval was not taken due to in vitro design of the study.

Informed Consent: N/A.

Author Contributions: Concept: M.G., K.S.; Design: K.S., A.A.; Supervision: K.S.; Resource: K.S., E.G.; Materials: E.G., M.G.; Data Collection and/ or Processing: E.G., M.G., A.A.; Analysis and/or Interpretation: A.A., E.G., K.S.; Literature Search: E.G., M.G.; Writing; E.G., M.G.; Critical Reviews: K.S., M.G., A.S., E.G.

Conflict of Interest: The authors have no conflicts of interest to declare.

Financial Disclosure: The authors declared that this study has received no financial support.

\section{REFERENCES}

I. Ekrem K, Rokan DK. Antibiotic susceptibility patterns of Pseudomonas aeruginosa strains isolated from various clinical specimens. Sky Journal of Microbiology Research Vol 20l4; 2(2): 13-7.

2. Fazeli H, Akbar R, Moghim, S, Nariman TB, Arabestan RA, Ghoddousi AR. Pseudomonas aeruginosa infections in patients, hospital means, and personnel's specimens. J Res Med Sci 2012; 17(4): 332-7.

3. Lister PD, Wolter DJ, Hanson ND. Antibacterial-resistant Pseudomonas aeruginosa: clinical impact and complex regulation of chromosomally encoded resistance mechanisms. Clin Microbiol Rev 2009; 22(4): 582-610. [CrossRef]

4. Shaikh S, Fatima J, Shakil S, Danish Rizvi SM, Kamal MA. Prevalence of multidrug resistant and extended spectrum beta-lactamase producing Pseudomonas aeruginosa in a tertiary care hospital. Saudi J Biol Sci 2015; 22(I): 62-4. [CrossRef]

5. Gaouar-Borsali N, Gauar-Yadi M, Babaahmed Z, Drissi M. Antibiotic resistance study of some clinical strains of Pseudomonas aeruginosa characterization by conjugation and cleaning out of plasmid. Der Pharma Chemica 2012; 4(3): II60-3.

6. Ak S, Yıldız F, Gündüz A, Köroğlu M. Pseudomonas aeruginosa Sușlarının Antibiyotiklere Duyarlııklarının Vitek 2 Otomatize Sistem ile Değerlendirilmesi. Gazi Med J 2016; 27: 62-4. [CrossRef]

7. Hirsch EB, Tam VH. Impact of multidrug-resistant Pseudomonas aeruginosa infection on patient outcomes. Expert Rev Pharmacoecon Outcomes Res 20I0; 10(4): 44I-5I. [CrossRef]

8. Paranjothi S, Dheepa R. Screening for multidrug resistance bacteria pseudomonas aeruginosa in hospitalized patient in hosur krishnagiri (DT). Int J Pharma Bio Sci 20I0; I(3): I-I5.

9. Öztürk CE, Albayrak HT, Altınöz A, Ankaralı H. Pseudomonas aeruginosa sușlarında antibiyotiklere direnç ve beta-laktamaz oranları. Ankem Derg 20I0; 24(3): II7-23.

10. Premalatha DE, Siddesh KC, Halesh LH, Koppad M, Prakash N. Antibiotic resistance pattern of Pseudomonas aeruginosa strains isolated from clinical specimens in a tertiary care hospital. Int J Recent Trends Sci Techn 2015; 13(3): 48I-3. 
II. Jafar K, Wahab A, Qayyum A, Jamshed S. Drug resistance pattern of Pseudomonas aeruginosa isolates at PIMS Hospital, Islamabad. J Chem Pharm Res 2014; 6(II): 715-9.

12. Al-Hasan MN, Wilson JW, Lahr BD, Eckel-Passow JE, Baddour LM. Incidence of Pseudomonas aeruginosa bacteremia: a population-based study. Am J Med 2008; I2I(8): 702-8. [CrossRef]

13. Aykan ȘB, Ciftçi iH. Changes in antibiotis resistance of Pseudomonas aeruginosa isoates over the past II years in turkey: a meta-analysis. Mikrobiyo Bul 2015; 49(3): 352-65. [CrossRef]
14. Ruh E, Gazi U, Güvenir M, Süer K, Çakır N. Antibiotic resistance rates of Pseudomonas aeruginosa, Acinetobacter baumanii and Klebsiella pneumonia isolated from a university-affiliated hospital in North Cyprus. Turk Hij Den Biyol Derg 2016; 73(4): 333-44. [CrossRef]

15. European Center for Disease Prevention and Control. Antimicrobial resistance surveillance in Europe 2015. Annual Report of the European Antimicrobial Resistance Surveillance Network (EARS-NET). 2015. Stockholm: ECDC. 